\title{
A One-Equation Turbulence Transport Model for High Reynolds Number Wall-Bounded Flows
}

Barrett S. Baldwin and Timothy J. Barth

August 1990

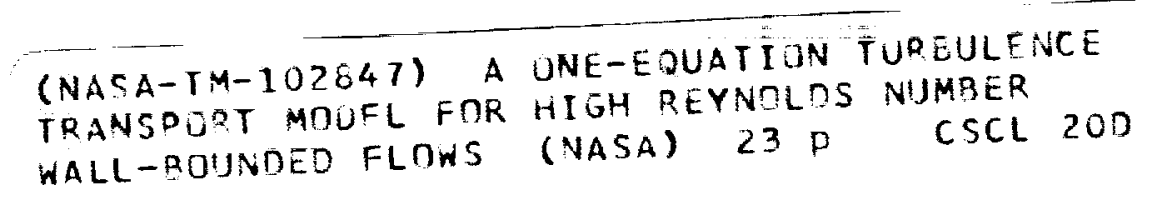

(NASA-TM-102847) WALL-BOUNDED FLOWS

HIGH REYNOLOS
(NASA) $23 \mathrm{p}$
NO1-10252

Unclas

$53 / 34 \quad 0310031$

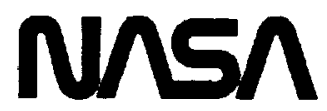

National Aeronautics and Space Administration 
$\checkmark$

5

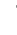


NASA Technical Memorandum 102847

\section{A One-Equation Turbulence Transport Model for High Reynolds Number Wall-Bounded Flows}

Barrett S. Baldwin and Timothy J. Barth, Ames Research Center, Moffett Field, California

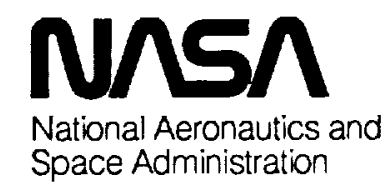



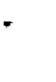

. 


\section{SUMMARY}

A one-equation turbulence model that avoids the need for an algebraic length scale is derived from a simplified form of the standard $k-\epsilon$ model equations. After calibration based on well established properties of the flow over a flat plate, predictions of several other flows are compared with experiment. The preliminary results presented indicate that the model has predictive and numerical properties of sufficient interest to merit further investigation and refinement. The one-equation model is also analyzed numerically and robust solution methods are presented.

\section{INTRODUCTION}

One motivation for the developments documented in this report was the inability of well-established Navier-Stokes solvers using algebraic turbulence models to adequately predict several of the turbulent flow fields contained in the Viscous Transonic Airfoil Workshopt. These flows contained significant separated flow and uniformly poor results were reported by all participants using the algebraic turbulence models of Baldwin-Lomax (ref. 1) or Cebeci-Smith (ref. 2). (The results predicted by the ARC2D code were reported by Maksymiuk and Pulliam in ref. 3.) Two cases were adequately predicted by only one turbulence model, that of Johnson and King (ref. 4) as reported by King (ref. 5) and verified by Coakley (ref. 6) using an independent code.

Another motivation is the need to treat flow problems in which multiple shear layers are present such that the determination of algebraic length scales is cumbersome and unreliable. An example is the Coanda airfoil configuration computed by Pulliam, Jespersen, and Barth (ref. 7) which exploits tangential surface blowing. The need to avoid algebraic length scales leads to a consideration of $k-\epsilon$ or related two-equation models.

From our limited experience with two-equation models and reports by others (e.g., Sugavanum (ref. 8)) it became apparent that it would be worthwhile to investigate the possibility of transforming to variables other than the basic physical variables in an effort to avoid the well-known numerical difficulties that occur in the solution of the standard $k-\epsilon$ equations. In the course of that investigation, a self-consistent one-equation model was found that also avoids the need for algebraic length scales. The main purpose of this report is to present the one-equation model and show the applicability of the model to a range of difficult turbulent flows. Results from computations of the two troublesome cases in the Viscous Transonic Airfoil Workshop are reported and significant improvement is achieved.

The following two sections explain the rationale behind the development of the oneequation model. The next contains the solution for a self-similar turbulent wake to demonstrate a degree of generality of the model. In the overall design of the model equation, emphasis was placed on numerical considerations so that extremely robust numerical solution methods could be used. The Numerical Implementation section gives some of the numerical theory and analysis needed to properly incorporate the one-equation model into flow solvers. The one-equation model has been implemented in a number of central-difference

$\dagger$ Held in conjunction with the AIAA 25th Aerospace Sciences Meeting (January 1987). 
and upwind finite-volume Navier-Stokes solvers in both two and three space dimensions and generalized coordinates. The computer code for these implementations can be obtained by contacting the second author (barth@prandtl.nas.nasa.gov). Finally in the appendix, we completely summarize the one-equation model for compressible flow.

The authors are grateful to Drs. P.R. Spalart and T.J. Coakley for useful discussions and for reviewing the report.

\section{DERIVATION OF THE $k-R_{T}$ MODEL}

We begin with a standard form of the $k-\epsilon$ equations (see Patel et al. (ref. 9)):

$$
\begin{aligned}
& \frac{D k}{D t}=\nabla \cdot\left(\nu+\frac{\nu_{t}}{\sigma_{k}}\right) \nabla k+P-\epsilon \\
& \frac{D \epsilon}{D t}=\nabla \cdot\left(\nu+\frac{\nu_{t}}{\sigma_{\epsilon}}\right) \nabla \epsilon+c_{\epsilon_{1}} \frac{\epsilon}{k} P-c_{\epsilon_{2}} \frac{\epsilon^{2}}{k}
\end{aligned}
$$

where $\frac{D}{D t}$ denotes the substantive derivative, $\frac{D}{D t}=\frac{\theta}{\partial t}+\mathbf{V} \cdot \nabla$ and $P$ the production of $k, P=\nu_{t}\left(\frac{\partial U_{i}}{\partial x_{j}}+\frac{\partial U_{j}}{\partial x_{i}}\right) \frac{\partial U_{i}}{\partial x_{j}}-\frac{2}{3} \nu_{t}\left(\frac{\partial U_{k}}{\partial x_{k}}\right)^{2}$. From these two equations we are free to form a third by considering linear and nonlinear combinations. In our case, we do this to form equivalent systems which have improved numerical properties. We will return to this discussion in a later section. In particular, we consider a field equation for the "turbulence Reynolds number," $R_{T}$

$$
R_{T}=\frac{k^{2}}{\nu \epsilon} \quad \text { (turbulence Reynolds number) }
$$

The $R_{T}$ field equation is obtained from the $k-\epsilon$ equations by considering differentials of $R_{T}, d R_{T} / R_{T}=2 d k / k-d \epsilon / \epsilon$. It should be clear that the substantive derivatives of $k$ and $\epsilon$ as well as their respective source terms transform without approximation. In transforming the diffusion terms (which are modeled in both the $k$ and $\epsilon$ equations), we omit certain terms arising from the transformation to obtain a new diffusion model for the $R_{T}$ equation.

$$
\frac{D\left(\nu R_{T}\right)}{D t}=\left(2-c_{\epsilon_{1}}\right) \frac{\nu R_{T}}{k} P+\left(c_{\epsilon_{2}}-2\right) k+\left(\nu+\frac{\nu_{t}}{\sigma_{\epsilon}}\right) \nabla^{2}\left(\nu R_{T}\right)-\frac{1}{\sigma_{\epsilon}}\left(\nabla \nu_{t}\right) \cdot \nabla\left(\nu R_{T}\right)
$$

where

$$
\nu_{t}=c_{\mu}\left(\nu R_{T}\right)
$$

Note that since $\nu_{t}$ should not depend on $\nu$ at large $R_{T}$, the appropriate field variable is $\nu R_{T}=k^{2} / \epsilon$ rather than $R_{T}$.

Equation (2) can be rearranged in the form

$$
\epsilon=\frac{k^{2}}{\nu R_{T}}=\frac{\left(k_{1}+k_{2}\right)^{2}}{\nu R_{T}}
$$


Without loss of generality, we can assign

$$
k_{1}^{2}=\nu R_{T} P \quad\left(\text { at large } \mathrm{R}_{\mathrm{T}}\right)
$$

In regions where $k_{2}<<k_{1}$ this will result in $P \approx \epsilon$. Note that the relation

$$
k^{2}=\left(k_{1}+k_{2}\right)^{2}=k_{1}^{2}\left(1+\frac{k_{2}}{k_{1}}\right)^{2}=\nu R_{T} P\left(1+\frac{k_{2}}{k_{1}}\right)^{2}
$$

or

$$
k=\sqrt{\nu R_{T} P}\left(1+\frac{k_{2}}{k_{1}}\right)
$$

is still completely general. Substitution of equation (6) into equation (3) and rearrangement leads to

$$
\begin{aligned}
\frac{D\left(\nu R_{T}\right)}{D t} & =\left(c_{\epsilon_{2}}-c_{\epsilon_{1}}\right) \sqrt{\nu R_{T} P}+\left(\nu+\frac{\nu_{t}}{\sigma_{\epsilon}}\right) \nabla^{2}\left(\nu R_{T}\right)-\frac{1}{\sigma_{\epsilon}}\left(\nabla \nu_{t}\right) \cdot \nabla\left(\nu R_{T}\right) \\
& -\left(2-c_{\epsilon_{1}}\right) \frac{k_{2}}{k_{1}+k_{2}} \sqrt{\nu R_{T} P}-\left(2-c_{\epsilon_{2}}\right) k_{2}
\end{aligned}
$$

The systei. 1 can be closed by substituting equation (6) in the $k$ equation to obtain

$$
\frac{D k}{D t}=-2 \sqrt{\frac{P}{\nu R_{T}}} k_{2}-\frac{k_{2}^{2}}{\nu R_{T}}+\nabla \cdot\left(\nu+\frac{\nu_{t}}{\sigma_{k}}\right) \nabla k
$$

$k_{2}=k-\sqrt{\nu R_{T} P}$.

Use of equations (4) and (5) requires further comment. These steps can be viewed as a means for exploiting the approximation resulting from equating production to dissipation, but in a way that rational procedures for departures from that approximation remain available.

\section{THE ONE-EQUATION $R_{T}$ MODEL FOR WALL-BOUNDED FLOWS}

It is of interest to note that by neglecting the last two terms in equation (7), a self-consistent one-equation model is obtained that should be a valid approximation over a major portion of shear layers. The main objective of this report is to develop modifications to this one-equation model that will allow it to be used in all parts of a shear layer. For that purpose, all of the previous relations are taken to be applicable at sufficiently large $R_{T}$. To arrive at a model that is applicable in near-wall regions, the turbulence Reynolds number $R_{T}$ is split into two factors

$$
R_{T}=\widetilde{R}_{T} f_{3}\left(\tilde{R}_{T}\right)
$$

where $f_{3}$ is a damping function such that $R_{T} \approx \widetilde{R}_{T}$ at large $R_{T}$. In addition, damping functions commonly used in $k-\epsilon$ models (ref. 9) are introduced so that

$$
\nu_{t}=\nu c_{\mu} f_{\mu} R_{T}=\nu c_{\mu} f_{\mu} f_{3} \tilde{R}_{T}
$$


and

$$
\epsilon^{\prime}=\epsilon-D=\frac{k^{2}}{\nu R_{T}}=\frac{\left(k_{1}+k_{2}\right)^{2}}{\nu f_{3} \widetilde{R}_{T}}
$$

The definition of $k_{1}$ applicable at small $R_{T}$ (and at all $R_{T}$ ) is taken to be

$$
k_{1}^{2}=\nu \widetilde{R}_{T} P
$$

This will allow $k_{1}$ to be the dominant part of $k$ in the near-wall region if the damping function $f_{3}$ is designed to accomplish that purpose. The resulting field equation for $\vec{R}_{T}$ is

$$
\frac{D\left(\nu \widetilde{R}_{T}\right)}{D t}=\left(c_{\epsilon_{2}} f_{2}-c_{\epsilon_{1}}\right) \sqrt{\nu \widetilde{R}_{T} P}+\left(\nu+\frac{\nu_{t}}{\sigma_{\epsilon}}\right) \nabla^{2}\left(\nu \widetilde{R}_{T}\right)-\frac{1}{\sigma_{\epsilon}}\left(\nabla \nu_{t}\right) \cdot \nabla\left(\nu \widetilde{R}_{T}\right)
$$

Incompressible flow over a flat plate with zero pressure gradient is used to determine suitable damping functions and to help calibrate the model. The thin shear layer approximation is used so that the production $P$ is approximated by

$$
P=\nu_{t}\left(u_{y}\right)^{2} \quad \text { (thin shear layer assumption) }
$$

Then

$$
\nu \widetilde{R}_{T} P=c_{\mu}\left(\nu \widetilde{R}_{T} u_{y}\right)^{2} f_{\mu} f_{3}
$$

and the model equation reduces to

$$
\frac{D \widetilde{R}_{T}}{D t}=\left(c_{\epsilon_{2}} f_{2}-c_{\epsilon_{1}}\right) \sqrt{c_{\mu} f_{\mu} f_{3}} \widetilde{R}_{T} u_{y}+\left(\nu+\frac{\nu_{t}}{\sigma_{\epsilon}}\right)\left(\widetilde{R}_{T}\right)_{y y}-\frac{1}{\sigma_{\epsilon}}\left(\nu_{t}\right)_{y}\left(\widetilde{R}_{T}\right)_{y}
$$

At sufficiently high momentum thickness Reynolds number the beginning of the $\log$ layer occurs in a region where the total shear stress is approximately constant and equal to the shear stress at the wall. In the log region and below, where advective terms are negligible, the $x$-momentum equation becomes simply

$$
\left(\nu+\nu_{t}\right) u_{y}=u_{\tau}^{2} \quad(\log \text { region and below })
$$

where $u_{\tau}$ is the friction velocity $\sqrt{\tau_{\text {wall }} / \rho_{\text {wall }}}$. In the $\log$ region where $u_{y}=u_{\tau} /(\kappa y)$, $\nu<<\nu_{t}$, and $\nu_{t}=\nu c_{\mu} \widetilde{R}_{T}$ we have

$$
\nu_{t}=\frac{u_{\tau}^{2}}{u_{y}}=\kappa u_{\tau} y \quad(\log \text { region })
$$

where $\kappa$ is the Karman constant and

$$
\widetilde{R}_{T}=\frac{\nu_{t}}{\nu c_{\mu}}=\frac{\kappa}{c_{\mu}} \frac{u_{\tau} y}{\nu} \quad \text { (log region) }
$$

In this case $\left(\widetilde{R}_{T}\right)_{y y}=0$ and substitution in equation (15) with damping functions set to unity and advection terms zero produces the following formula:

$$
\frac{1}{\sigma_{\epsilon}}=\left(c_{\epsilon_{2}}-c_{\epsilon_{1}}\right) \sqrt{c_{\mu}} / \kappa^{2}
$$


In the region below the log layer an additional relation is needed to determine the definitions of $\widetilde{R}_{T}$ and $f_{3}\left(\widetilde{R}_{T}\right)$. After a study of the consequences, we have imposed

$$
\widetilde{R}_{T}=\frac{\kappa}{c_{\mu}} \frac{u_{\tau} y}{\nu} \quad \text { (log region and below) }
$$

We can ensure that this relation is consistent with the field equation for $\widetilde{R}_{T}$ (eqn. (15)) by requiring that the damping function $f_{2}\left(y^{+}\right)$be adjusted to accomplish that purpose. By a procedure that will be described, the following damping functions have been determined:

$$
\begin{aligned}
f_{\mu} & =D_{1} / D_{3} \\
f_{3} & =D_{2} D_{3}
\end{aligned}
$$

where

$$
\begin{gathered}
D_{1}=1-\exp \left(-y^{+} / A^{+}\right), \quad A^{+}=26 \\
D_{2}=1-\exp \left(-y^{+} / A_{2}^{+}\right), \quad A_{2}^{+}=10 \\
D_{3}=1+B_{3} \exp \left(-y^{+} / A_{3}^{+}\right)\left[1-\exp \left(-y^{+} / A_{3}^{+}\right)\right], \quad B_{3}=5.2, A_{3}^{+}=15
\end{gathered}
$$

where $y^{+}=u_{\tau} y / \nu$. From these expressions we have that $f_{\mu} f_{3}=D_{1} D_{2}$. It should be emphasized that neither $f_{\mu}$ nor $f_{3}$ is equal to $D_{1}$ or $D_{2}$. However, it will be seen that implementation of the one-equation model requires only the evaluation of the product $f_{\mu} f_{3}$ and does not require knowledge of $f_{\mu}$ and $f_{3}$ individually. Since $D_{1}$ and $D_{2}$ are simpler functions than $f_{\mu}$ and $f_{3}$, it is convenient to replace the product $f_{\mu} f_{3}$ with $D_{1} D_{2}$ wherever it occurs.

Substitution of equations (17) and (18) in equation (15) with $D \widetilde{R}_{T} / D t=0$ yields

$$
\left(c_{\epsilon_{2}} f_{2}-c_{\epsilon_{1}}\right) \sqrt{c_{\mu} D_{1} D_{2}} y u_{y}=\left(c_{\epsilon_{2}}-c_{\epsilon_{1}}\right) \frac{\sqrt{c_{\mu}}}{\kappa^{2}}\left(\nu_{t}\right)_{y}
$$

Substitution of equation (16) to remove $u_{y}$ and equations (10) and (18) to remove $\nu_{t}$ produces

$$
\begin{aligned}
f_{2}\left(y^{+}\right)=\frac{c_{\epsilon_{1}}}{c_{\epsilon_{2}}} & +\left(1-\frac{c_{\epsilon_{1}}}{c_{\epsilon_{2}}}\right)\left(\frac{1}{\kappa y^{+}}+D_{1} D_{2}\right)\left(\sqrt{D_{1} D_{2}}\right. \\
& \left.+\frac{y^{+}}{\sqrt{D_{1} D_{2}}}\left(\frac{1}{A^{+}} \exp \left(-y^{+} / A^{+}\right) D_{2}+\frac{1}{A_{2}^{+}} \exp \left(-y^{+} / A_{2}^{+}\right) D_{1}\right)\right)
\end{aligned}
$$

For small $y^{+}$we have the following limit value:

$$
\lim _{y^{+} \rightarrow 0} f_{2}\left(y^{+}\right)=\frac{c_{\epsilon_{1}}}{c_{\epsilon_{2}}}+\left(1-\frac{c_{\epsilon_{1}}}{c_{\epsilon_{2}}}\right) \frac{3}{\kappa \sqrt{A^{+} A_{2}^{+}}}
$$

After assignment of the parameters $c_{\epsilon_{1}}, c_{\epsilon_{2}}, \kappa$, equation (19) can be used to determine the damping function $f_{2}\left(y^{+}\right)$when the combination $f_{\mu} f_{3}=D_{1} D_{2}$ is known as a function of $y^{+}$. For our choice of constants we have a limit value of $f_{2}(0) \approx 0.781$. 
From equation (18) it is seen that $y^{+}$in the damping functions can be replaced by

$$
y^{+}=\frac{c_{\mu}}{\kappa} \widetilde{R}_{T} \quad\left(\text { when } p_{x}=0\right)
$$

so that the damping functions can be expressed in terms of $\widetilde{R}_{T}$ or (implicitly) in terms of the turbulence Reynolds number $R_{T}=\widetilde{R}_{T} f_{3}\left(\widetilde{R}_{T}\right)$. For general flows that involve nonzero pressure gradients, the dependence on $\widetilde{R}_{T}$ or $y^{+}$is not interchangeable. Experience will determine whether the functional dependence on $\widetilde{R}_{T}$ or $y^{+}$is preferable.

In this report, for simplicity, we have adhered to dependence on $y^{+}$, but we do not recommend this as a final choice. We have calibrated these functions by comparison with results from the (inner) Cebeci-Smith model (ref. 2) using equation (16). The resulting prediction of $\nu_{t}$ from that model in the $\log$ region and below is

$$
\left(\nu_{t}\right)_{C S}=\frac{\nu}{2}\left[\sqrt{1+\left(4 \kappa y^{+} D_{1}\right)^{2}}-1\right]
$$

For the present model, substitution of equation (20) in equation (10) yields

$$
\nu_{t}=\nu f_{\mu} f_{3} \kappa y^{+}=\nu D_{1} D_{2} \kappa y^{+}
$$

For either model $u^{+}$is obtained by the integration of equation (16):

$$
u^{+}=\int_{0}^{y^{+}} \frac{d y^{+}}{1+\nu_{t} / \nu}
$$

Figure 1 shows a comparison of the predicted variations of $\nu_{t} / \nu$ and $u^{+}$. The close agreement of the two models is not surprising because the damping functions $D_{1}$ and $D_{2}$ were designed for that purpose.

From equations (10), (12), (14), (16), and (18) explicit formulas for $k_{1}^{+}$and $P^{+}$in the inner region are obtained

$$
\begin{array}{ll}
k_{1}^{+}=\frac{1}{\sqrt{c_{\mu}} \frac{\sqrt{D_{1} D_{2}} \kappa y^{+}}{1+D_{1} D_{2} \kappa y^{+}}} \quad \text { (log region and below) } \\
P^{+}=\frac{\kappa y^{+} D_{1} D_{2}}{\left(1+\kappa y^{+} D_{1} D_{2}\right)^{2}} \quad \text { (log region and below) }
\end{array}
$$

A plot of the $k_{1}^{+}$variation is shown in Figure 2. It is somewhat surprising that $k_{1}$ provides a more realistic turbulent energy distribution than several $k-\epsilon$ models, although no attention was paid to that purpose in the design of $D_{1}$ and $D_{2}$. 


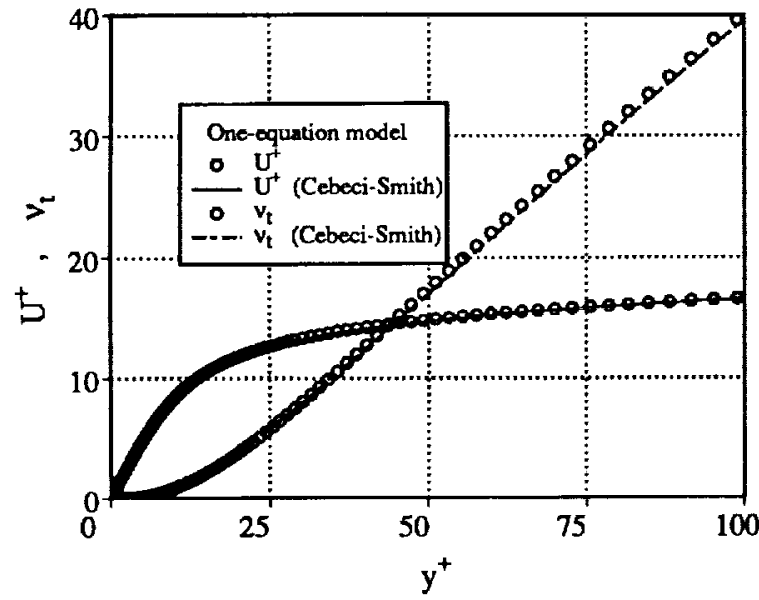

Figure 1. Comparison of $U^{+}$and $\nu_{t}$ between Cebeci-Smith and the oneequation model.

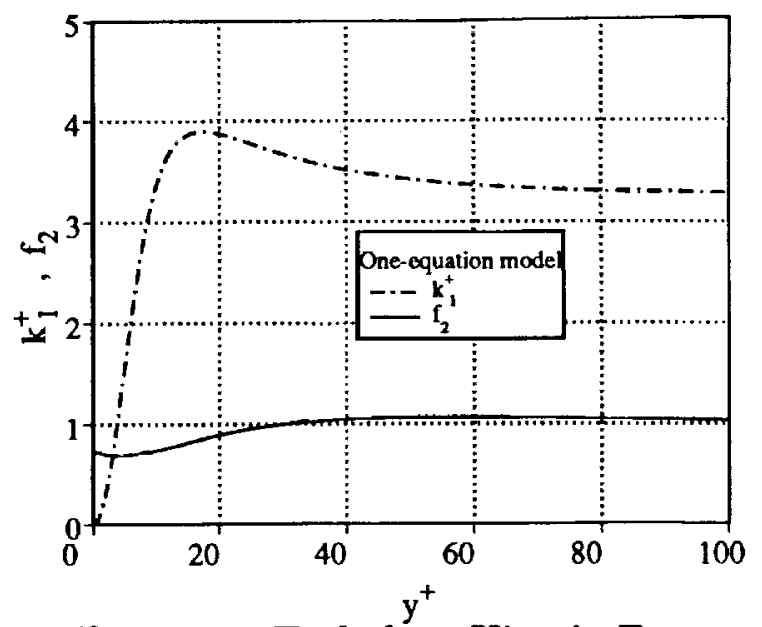

Figure 2. Turbulent Kinetic Energy and damping function $\left(f_{2}\right)$ distributions.

Since $f_{3}$ has been introduced, it seems necessary that it be fully defined. The above $D_{3}$ function was calibrated to produce a reasonable variation of $\epsilon$ in the near-wall region, although neither $\epsilon$ nor $D_{3}$ is needed in calculations based on our one-equation model. To complete the definition of $\widetilde{R}_{T}$ and $R_{T}$, the quantity $D$ in equation (11) is taken to be $\nu k_{y y}$ (from Mansour, Kim, and Moin (ref. 10)).

For the sake of completeness, plots of $R_{T}, \widetilde{R}_{T}, f_{3}, f_{\mu}$ and $\epsilon$ are shown in figures 3-5, although, as mentioned earlier, they are not needed for implementation of our model. The $\epsilon$ distribution was evaluated assuming that $k \approx k_{1}$, (i.e., $k_{2}<<k_{1}$ ) in the log region and below. The damping function $D_{3}$ was designed to make the $\epsilon$ distribution resemble that found from the direct simulations reported in reference 10 . However the resulting rather realistic $f_{\mu}$ distribution is a byproduct that was not anticipated.

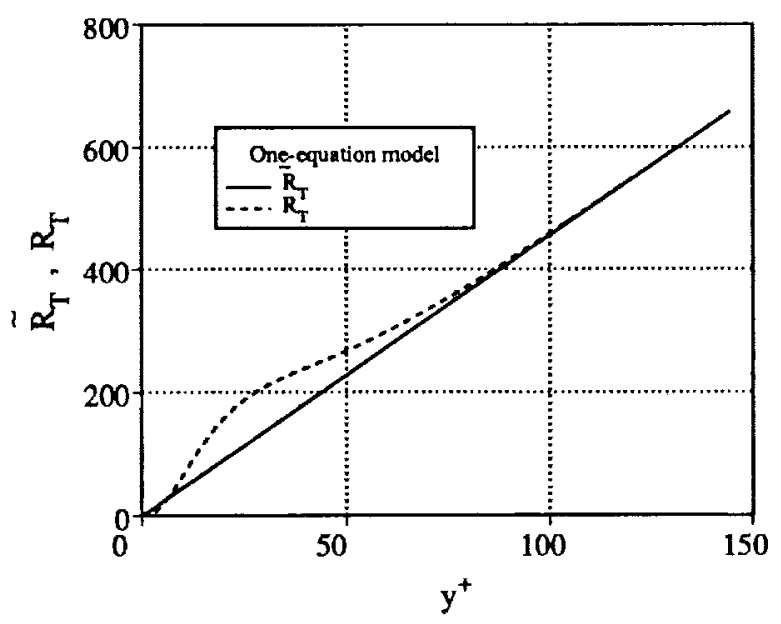

Figure 3. $\widetilde{R}_{T}, R_{T}$ distributions.

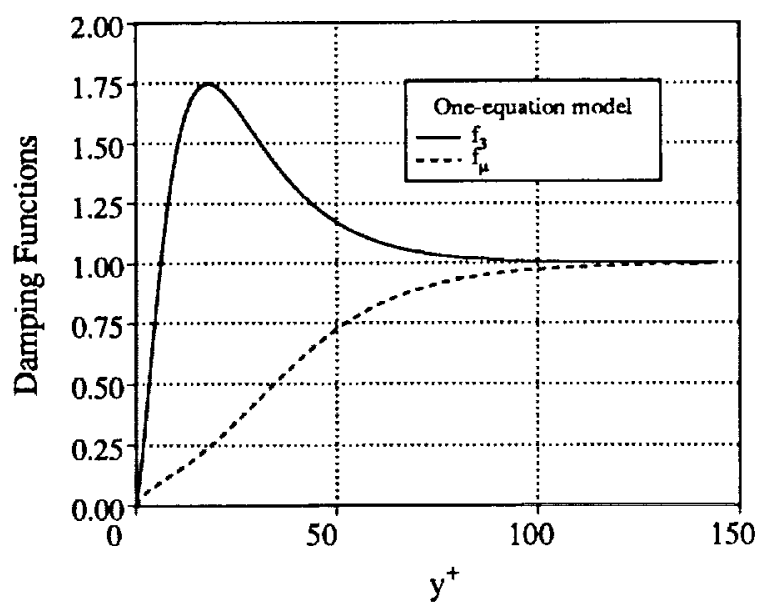

Figure 4. Damping functions. 


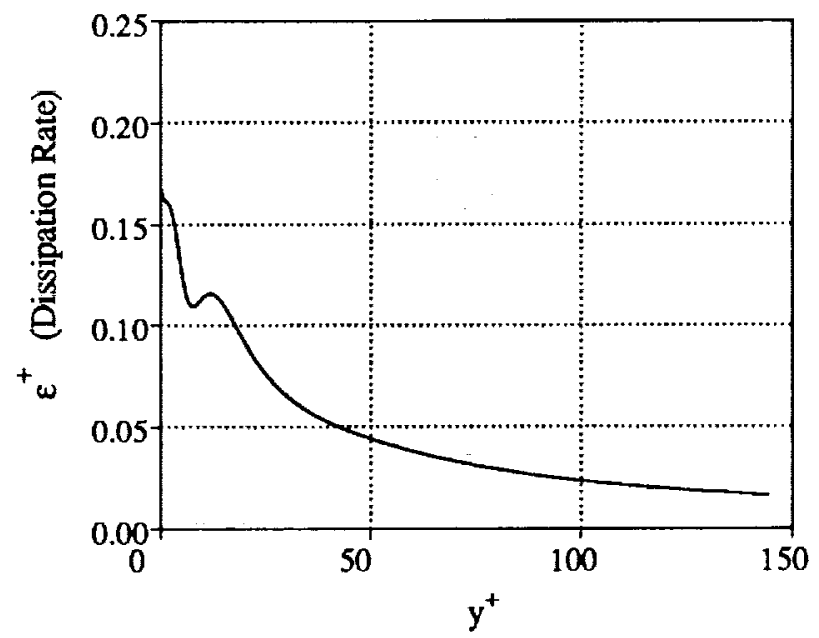

Figure 5. Near-wall epsilon distribution.

To complete the calibration of the one-equation model, values of the parameters $\kappa, c_{\mu}, c_{\epsilon_{1}}, c_{\epsilon_{2}}$ are needed. In this report we have adopted the widely used values $\kappa=$ $0.41, c_{\mu}=0.09$ and set $c_{\epsilon_{2}}=2.0$, so that the last term in equation (7) will be identically zero. We have adjusted $c_{\varepsilon_{1}}$ to match the calculated skin friction coefficient for incompressible flow over a flat plate to a compilation of experimental data that is well represented by the Karman-Schoenherr formula given in Hopkins and Inouye (ref. 11):

$$
\frac{1}{C_{f}}=17.08\left(\log _{10} R e_{\theta}\right)^{2}+25.11 \log _{10} R e_{\theta}+6.012 \quad(\text { Karman }- \text { Schoenherr formula })
$$

The value of $c_{\epsilon_{1}}$ currently used is 1.2 , which is well below the "standard" value of 1.44 but is not the lowest on record, see reference 9 . An alternative to our low value of $c_{\epsilon_{1}}$ would be to impose a relatively large value of $\widetilde{R}_{T}$ in the outer flow. From equation 13 it can be seen that a constant value of $\widetilde{R}_{T}$ is self-preserving in regions where production is zero (unlike $k$ and $\epsilon$ in the $k-\epsilon$ equations). However, in this report, we adhere to $c_{\varepsilon_{1}}=1.2$ and a low level of $\widetilde{R}_{T}$ in the free stream such that $\nu_{t}$ in the free stream (and at the leading edge of an airfoil) will not be large compared to the molecular viscosity. 


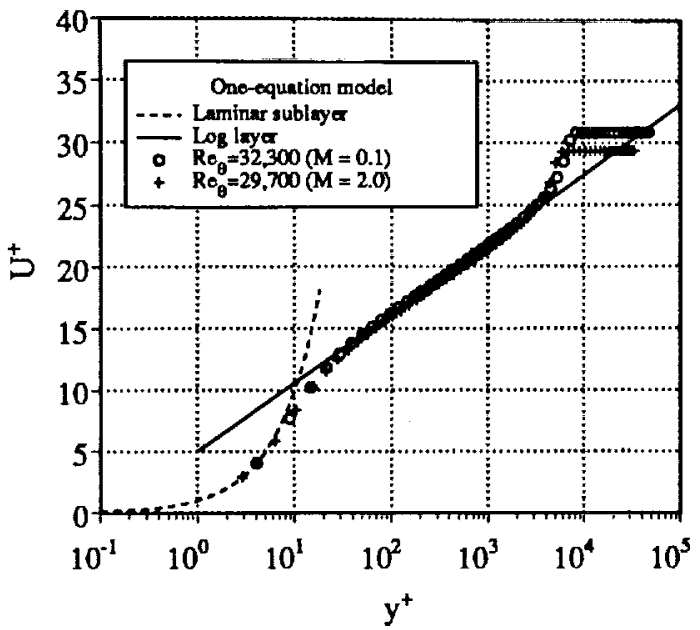

Figure 6. Law-of-the-wall.

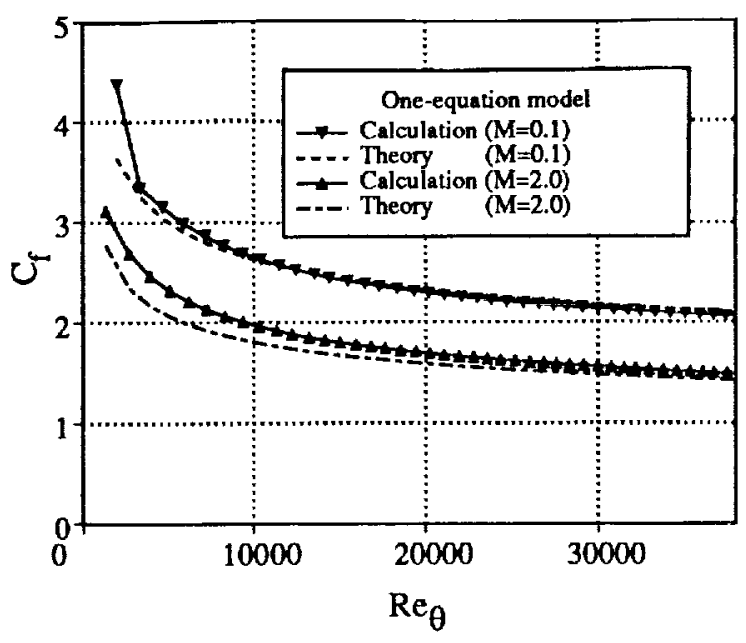

Figure 7. Skin friction on flat plate.

In this report, we extend the model to compressible flow following a practice which has been used for algebraic models (ref. 2). We assume that all the above relations apply to compressible flow with a temperature-dependent $\nu$ and with

$$
\mu_{t}=\rho \nu_{t}
$$

Figure 6 graphs computed law-of-the-wall solutions $\left(u^{+}=u^{*} / u_{\tau}\right.$ versus $\left.y^{+}\right)$for subsonic and supersonic Mach numbers where $u^{*}$ is defined (see, for example, Rubesin and Horstman (ref. 12)):

$$
u^{*}=\int_{0}^{U} \sqrt{\rho / \rho_{\text {wall }}} d U
$$

For incompressible flow this plot corresponds to the conventional law of the wall. The paper by Hopkins and Inouye (ref. 11) contains several alternative procedures for applying the Karman-Schoenherr formula to compressible flow. In the procedure by Sommer and Short an empirically determined temperature $T_{s s}$ is defined:

$$
T_{s s}=T_{e d g e}\left(1+0.035 M_{e d g e}^{2}\right)+0.45\left(T_{w a l l}-T_{e d g e}\right)
$$

The momentum thickness Reynolds number $R e_{\theta}$ is then adjusted to a corresponding incompressible value $\overline{R e}_{\theta}$ :

$$
\overline{\operatorname{Re}}_{\theta}=R e_{\theta} \mu\left(T_{\text {edge }}\right) / \mu\left(T_{s s}\right)
$$

Finally, the resulting $\bar{C}_{f}$ from the Karman-Schoenherr formula is adjusted to the compressible value by multiplying by the temperature ratio $T_{\text {edge }} / T_{s s}$. Figure 7 compares skin friction for compressible flow over a flat plate with the foregoing "theory" at subsonic and supersonic Mach numbers. The agreement for both subsonic and supersonic Mach numbers is good. The excellent agreement at low Mach numbers was expected because the model was calibrated in the incompressible limit.

Figure 8 contains law-of-the-wall plots for flat plate boundary-layer flow computed using several values of free-stream $\widetilde{R}_{T}$. Note that $\nu_{t} / \nu=c_{\mu} R_{T}=0.09 R_{T}$ when damping 
functions are at unity. The plots indicate insensitivity to free-stream values for all values of free stream $\widetilde{R}_{T}$ tested, except $\left(\widetilde{R}_{T}\right)_{\infty}=100$ which is the only value at which $\nu_{t} / \nu$ exceeds unity in the free stream.

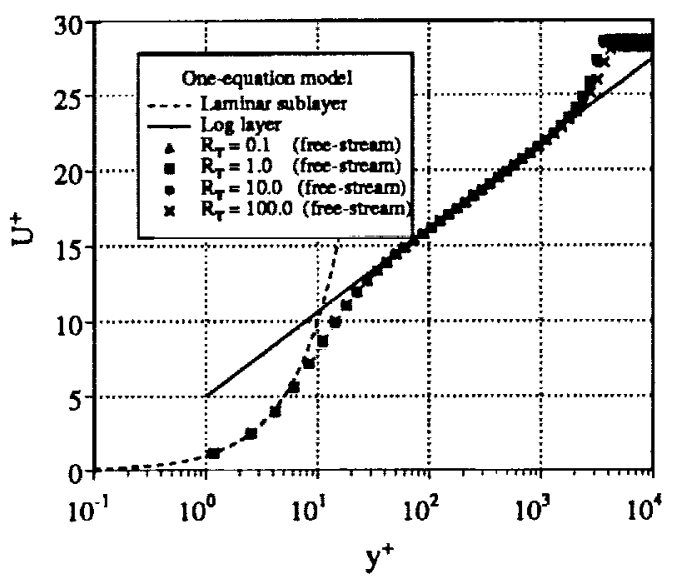

Figure 8. Sensitivity of boundary layer to values of free-stream $\widetilde{R}_{T}$.

To further assess the performance of the one-equation model, computations were performed for two of the troublesome cases in the Viscous Transonic Airfoil Workshop. Figures 9-11 show the mesh and solution for viscous flow over the RAE 2822 airfoil geometry at $M_{\infty}=0.75, \alpha=2.72^{\circ}$, and a Reynolds number of 6.2 million. Computed lift and drag coefficients for the one-equation model were $C_{L}=0.771, C_{D}=0.0352$. This is compared with the Baldwin-Lomax solution (also plotted) which produced lift and drag coefficients of $C_{L}=0.895, C_{D}=0.0279$. The shock position is substantially improved and the overall pressure coefficient distribution is in much better agreement with experiment.

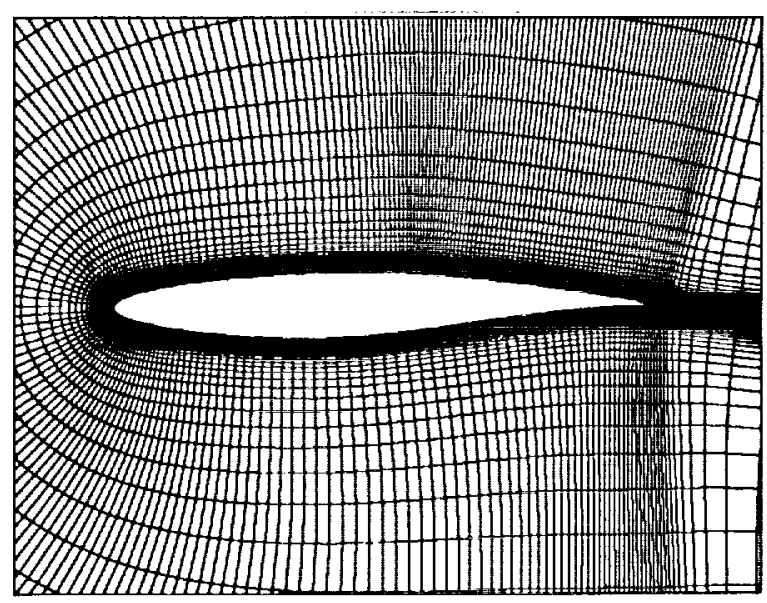

Figure 9. RAE 2822 mesh.

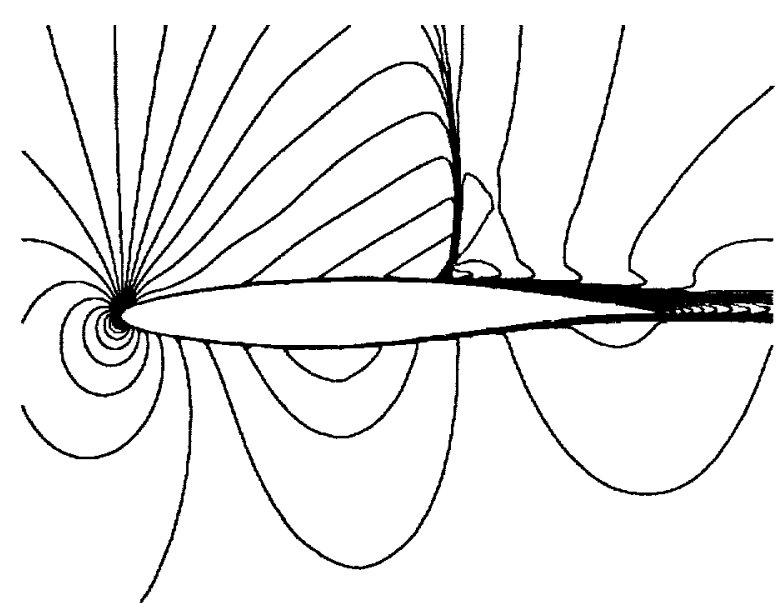

Figure 10. Mach contours $\left(M_{\infty}=\right.$ $0.75)$. 


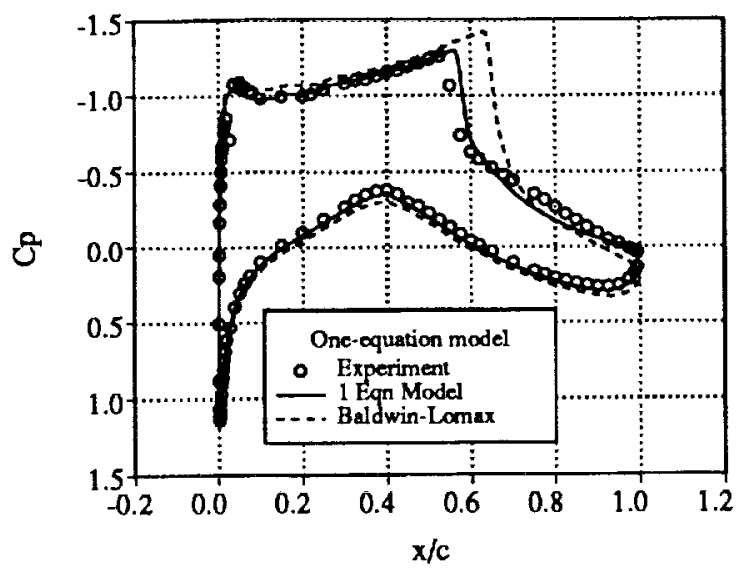

Figure 11. Pressure coefficient comparison.

The ext geometry and flow conditions provide a much more severe test case for turbulence modeling. The geometry is the standard NACA 0012 computed at $M_{\infty}=0.799$, $\alpha=2.26^{\circ}$, and a Reynolds number of 9 million. Figures $12-14$ show the grid and solution at these flow conditions. Figure 14 plots $C_{p}$ distributions for the one-equation model $\left(C_{L}=0.340, C_{D}=0.035\right)$, the Baldwin-Lomax model $\left(C_{L}=0.531, C_{D}=0.048\right)$, and the one-equation model with advection removed from the model equation on upper-surface flow only $\left(C_{L}=0.589, C_{D}=0.048\right)$.

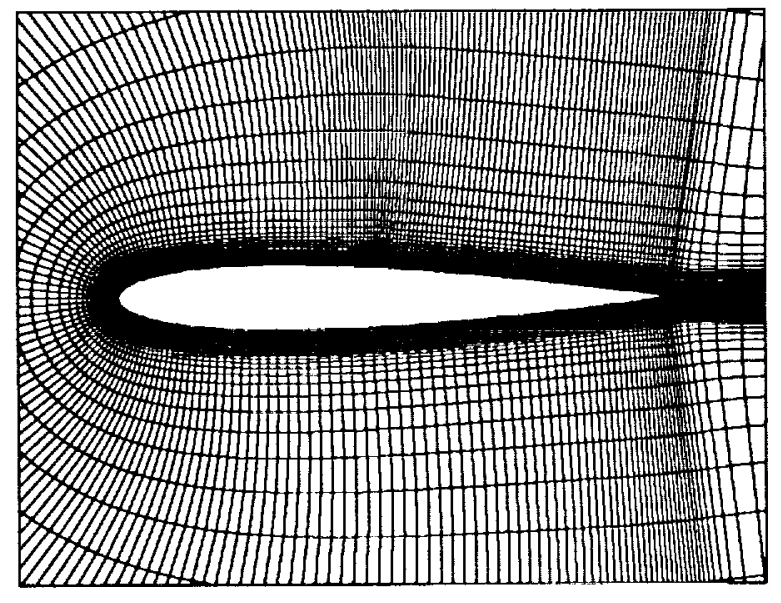

Figure 12. NACA 0012 mesh.

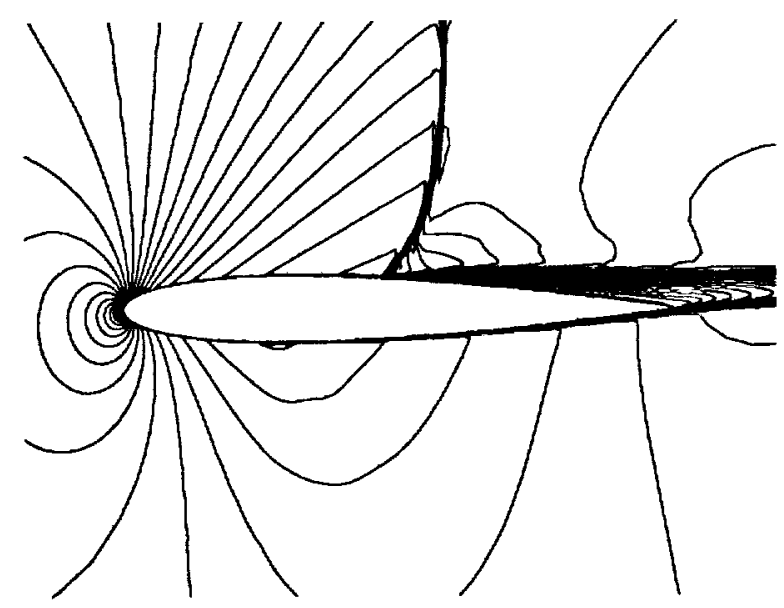

Figure 13. Mach contours $\left(M_{\infty}=\right.$ $0.8)$. 


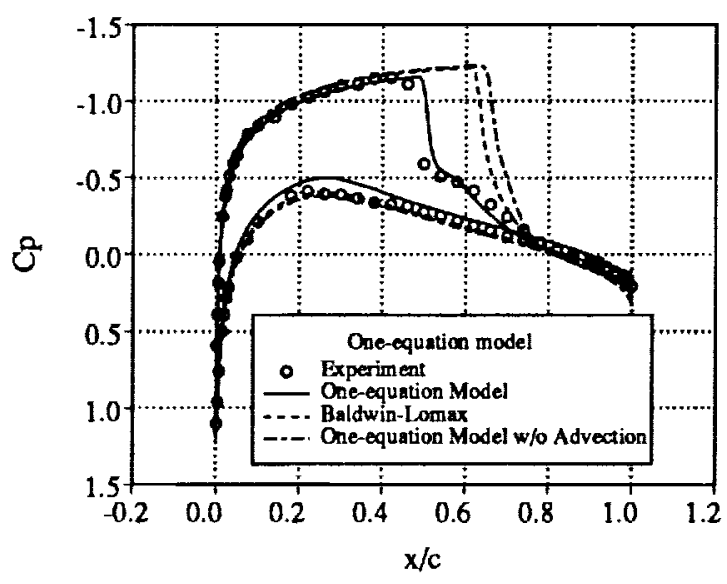

Figure 14. Pressure coefficient comparison.

The improvement in the full one-equation model is dramatic. The upper-surface shock wave is moved forward almost 20 percent chord and is in good agreement with experiment. The solution obtained with and without upper-surface advection clearly indicates the importance of upstream influences obtained via advective terms in this separated flow. Note that we have used the corrected angle of attack suggested by the experimenters $\left(\alpha_{\exp }=2.86^{\circ}\right)$. The discrepancy in lower-surface pressure and the effect of upper-surface shock location seems to indicate that this angle-of-attack correction does not adequately account for wind tunnel wall interference.

\section{THE ONE-EQUATION $R_{T}$ MODEL FOR FREE SHEAR LAYERS}

In this section we examine the one-equation $R_{T}$ model for the self-similar turbulent wake flow. Following the order-of-magnitude arguments of Tennekes and Lumley (ref. 13), the $x$ momentum equation for an eddy viscosity model calculation reduces to the following simplified form:

$$
U U_{x}=\left(\nu_{t} U_{y}\right)_{y}
$$

Using similar arguments, equation (7) reduces to

$$
\begin{aligned}
\bar{U}\left(\bar{R}_{T}\right)_{x} & =\left(c_{\epsilon 2}-c_{\epsilon 1}\right) \sqrt{\frac{R_{T} P}{\nu}}+\frac{1}{\sigma_{\epsilon}}\left[\nu_{t}\left(R_{T}\right)_{y y}-\left(\nu_{t}\right)_{y}\left(R_{T}\right)_{y}\right] \\
& -\left(2-c_{\epsilon 1}\right) \frac{k_{2}}{k_{1}+k_{2}} \sqrt{\frac{R_{T} P}{\nu}}-\left(2-c_{\epsilon 2}\right) \frac{k_{2}}{\nu}
\end{aligned}
$$

where $\nu_{t}=\nu c_{\mu} R_{T}, P=\nu_{t}\left(U_{y}\right)^{2}$, and $k_{1}=\sqrt{\frac{R_{X} P}{\nu}}$.

We are interested in the one-equation model that results from neglecting the last two terms of equation (22). However, it is worthwhile to gain an appreciation of how that approximation can apply more generally than in regions where production equals dissipation. Wake flows provide an interesting example. In this flow, production is zero 
at the centerline, but dissipation is not. Moving away from the centerline, the assumption that production is equal to dissipation becomes valid. Fortunately, even at the centerline of a wake flow we can rationalize neglecting the last two terms in equation (22). Clearly at the centerline of a wake the first of these two terms is identically zero because production vanishes there. Also note that many $k-\epsilon$ modelers choose $c_{\epsilon 2}=2$, which would remove the last term identically. The primary question still remains as to the overall validity of the one-equation approximation in a simple wake. Assuming self similarity of a turbulent wake, we can analyze the situation in detail. Following the scaling procedure of Tennekes and Lumley for wake flows, we set

$$
\begin{gathered}
U=U_{0}+U_{s} f(\xi) \\
R_{T}=\frac{U_{s} l}{\nu} h(\xi) \\
\nu_{t}=\nu c_{\mu} R_{T}=U_{s} l c_{\mu} h(\xi)
\end{gathered}
$$

where $\xi=y / l, U_{s}=A x^{-1 / 2}, l=B x^{1 / 2}$, and $U_{s}<<U_{0}$ are assumed. Substituting in equations (21) and (22) (with the last two terms of eqn. (22) omitted) we obtain

$$
\begin{gathered}
\beta\left(f+\xi f_{\xi}\right)+c_{\mu}\left(h f_{\xi}\right)_{\xi}=0 \\
\beta \xi h_{\xi}+\frac{c_{\mu}}{\sigma_{\epsilon}}\left[h h_{\xi \xi}-\left(h_{\xi}\right)^{2}\right]+\frac{\kappa^{2}}{\sigma_{\xi}}\left|f_{\xi}\right| h=0
\end{gathered}
$$

where

$$
\beta=\frac{U_{0} B}{2 A}=.08
$$

The numerical value of $\beta$ was evaluated from experiment by Tennekes and Lumley, based on the definitions $U_{s}=\left|U-U_{0}\right|_{\max }$, and by choosing a length $l$ as the distance from the centerline such that $\left|U-U_{0}\right| /\left|U-U_{0}\right|_{\max }=\exp (-1 / 2)$.

If $h(\xi)$ is a known function, equation (26) can be evaluated in the form

$$
f=\exp \left(\frac{-\beta}{c_{\mu}} \int_{0}^{\xi} \frac{\xi}{h} d \xi\right)
$$

This expression is then differentiated to replace $\left|f_{\xi}\right| h$ with $\beta \xi f / c_{\mu}$. Equation (27) can then be expressed in the following form:

$$
h_{\xi \xi}+\underbrace{\left(\frac{\beta_{1} \xi-h_{\xi}}{h}\right)}_{P_{1}(\xi)} h_{\xi}=\underbrace{-\left(\frac{\beta_{2} \xi f}{h}\right)}_{Q_{1}(\xi)}
$$

where $\beta_{1}=\frac{\beta \sigma_{\varepsilon}}{c_{\mu}}, \beta_{2}=\beta\left(\frac{\kappa}{c_{\mu}}\right)^{2}$. With $P_{1}$ and $Q_{1}$ considered known, the resulting equation is linear in $h_{\xi}$ and the solution is

$$
h_{\xi}=\int_{0}^{\xi} Q_{1}(\tilde{\xi}) \exp \left[P_{1 I}(\tilde{\xi})-P_{1 I}(\xi)\right] d \tilde{\xi}
$$


where $P_{1 I}=\int_{0}^{\xi} P_{1} d \xi$. By iterating the quadratures for $f$ and $h$, a numerical solution can be obtained in about 10 steps. In figure 15 we plot the solution of this equation and compare it with results from the constant eddy viscosity solution as well as results from experiment.

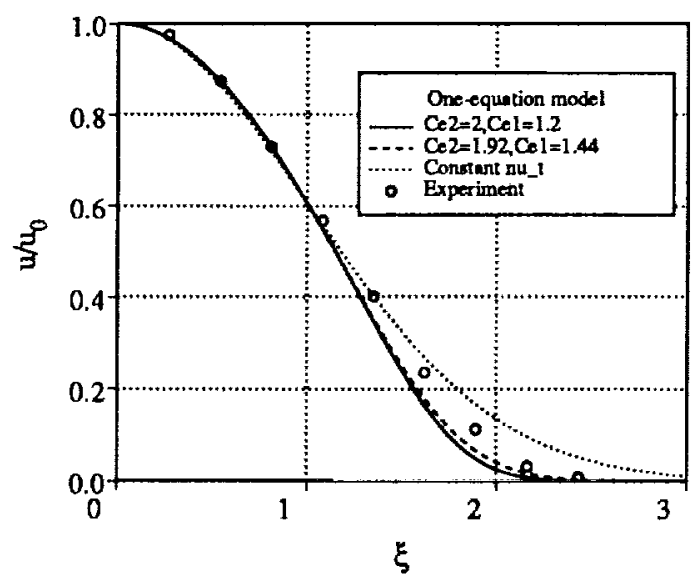

Figure 15. Comparison of experiment, and theory for self-similar wake flow.

The data points are from Townsend (ref. 14). The dashed curve was computed using our one-equation model with the values of $c_{\epsilon 1}$ and $c_{\epsilon 2}$ from the original "standard" $k-\epsilon$ model. The solid curve is based on the values of parameters that provide the best results for the wall-bounded flows considered in previous sections. The departure of the solid curve from the data is no worse than that of the dotted curve, which is from the widely used (in wakes) constant eddy viscosity approximation.

\section{NUMERICAL IMPLEMENTATION}

In this section we consider discretization of the one-equation model. We begin in a rather abstract setting by introducing the notion of positive operators for discrete systems. This will allow us to construct extremely robust algorithms for the one-equation model. Current implementations of the one-equation model in two- and three-space dimensions employ an implicit factored ADI solver for the scalar equation (decoupled from the mean flow equations). In the following analysis, implicit unfactored schemes will be considered. Good numerical behavior of this system is a prerequisite for the more general situation which might include matrix factorization.

We first define the solution vector $\mathcal{R}$ on a two-dimensional, logically rectangular mesh where $\mathcal{R}_{i j} \approx \nu \widetilde{R}_{T}\left(x_{i, j}, y_{i, j}\right)$ with

$$
\overrightarrow{\mathcal{R}}=\left[\mathcal{R}_{1,1}, \mathcal{R}_{1,2}, \ldots, \mathcal{R}_{1, M}, \mathcal{R}_{2,1}, \mathcal{R}_{2,2}, \ldots, \mathcal{R}_{2, M}, \ldots, \mathcal{R}_{N, 1}, \mathcal{R}_{N, 2}, \ldots, \mathcal{R}_{N, M}\right]^{T} .
$$

As we will see, our one-equation model with discretized advection and diffusion terms produces a system of ordinary differential equations of the form

$$
\overrightarrow{\mathcal{R}}_{t}+M(\overrightarrow{\mathcal{R}}) \overrightarrow{\mathcal{R}}=D \overrightarrow{\mathcal{R}}
$$


where $M(\overrightarrow{\mathcal{R}})$ is a matrix operator (possibly nonlinear) representing the discretization of advection and diffusion and $D$ is a diagonal matrix with positive entries representing the source term. We can construct implicit and explicit schemes of the form

$$
\left[I+\Delta t \theta M\left(\overrightarrow{\mathcal{R}}^{n}\right)\right]\left(\overrightarrow{\mathcal{R}}^{n+1}-\overrightarrow{\mathcal{R}}^{n}\right)=\Delta t\left(-M\left(\overrightarrow{\mathcal{R}}^{n}\right)+D\right) \overrightarrow{\mathcal{R}}^{n}
$$

or after rearrangement

$$
\left[I+\Delta t \theta M\left(\overrightarrow{\mathcal{R}}^{n}\right)\right] \overrightarrow{\mathcal{R}}^{n+1}=\left[I-(1-\theta) \Delta t M\left(\overrightarrow{\mathcal{R}}^{n}\right)+\Delta t D\right] \overrightarrow{\mathcal{R}}^{n}
$$

for all $\theta \in[0,1]$. As we will show, we can design numerical approximations for advection and diffusion which guarantee the following properties of $M\left(\overrightarrow{\mathcal{R}}^{n}\right)$ :

1. $M(\overrightarrow{\mathcal{R}})$ is a diagonally dominant monotone (M-type) matrix. M-type matrices are diagonally dominant matrices with positive diagonal entries and negative off-diagonal entries.

2. $M(\overrightarrow{\mathcal{R}})$ has zero row sum. This will be due to the use of the chain rule form of the equations

A well-known property of M-type matrices is that they have non-negative inverses, i.e., elements of the inverse are non-negative. We see from property 1 that the left-hand-side matrix of (33)

$$
\left[I+\Delta t \theta M\left(\overrightarrow{\mathcal{R}}^{n}\right)\right]
$$

is an M-type matrix and consequently

$$
\left[I+\Delta t \theta M\left(\overrightarrow{\mathcal{R}}^{n}\right)\right]^{-1} \geq 0, \quad \theta \geq 0
$$

The right-hand-side matrix of (33)

$$
\left[I-(1-\theta) \Delta t M\left(\overrightarrow{\mathcal{R}}^{n}\right)+\Delta t D\right]
$$

is also unconditionally non-negative for $\theta=1$ and non-negative under a CFL-like condition for $1>\theta \geq 0$. Assuming $\overrightarrow{\mathcal{R}}^{0} \geq 0$, from the solution update equation

$$
\overrightarrow{\mathcal{R}}^{n+1}=\left[I+\Delta t \theta M\left(\overrightarrow{\mathcal{R}}^{n}\right)\right]^{-1}\left[I-(1-\theta) \Delta t M\left(\overrightarrow{\mathcal{R}}^{n}\right)+\Delta t D\right] \overrightarrow{\mathcal{R}}^{n}
$$

positivity of $\overrightarrow{\mathcal{R}}^{n+1}$ is guaranteed whenever (34) is a nonnegative operator.

We now investigate stability properties of the numerical scheme. Given properties 1 and 2, it is a simple matter (see, for example, Barth and Lomax (ref. 15)) to show that

$$
\left\|\left[I+\Delta t \theta M\left(\overrightarrow{\mathcal{R}}^{n}\right)\right]^{-1}\right\|_{\infty} \leq 1
$$


We also have that

$$
\|[I-(1-\theta) \Delta t M(\overrightarrow{\mathcal{R}})]\|_{\infty} \leq 1
$$

under the CFL-like condition for positivity. If we first consider the system without the source term $(D=0)$ and Dirichlet boundary conditions, we have stability under the CFLlike condition for non-negativity

$$
\begin{aligned}
\left\|\overrightarrow{\mathcal{R}}^{n+1}\right\|_{\infty} & =\left\|\left[I+\Delta t \theta M\left(\overrightarrow{\mathcal{R}}^{n}\right)\right]^{-1}[I-(1-\theta) \Delta t M(\overrightarrow{\mathcal{R}})] \overrightarrow{\mathcal{R}}^{n}\right\|_{\infty} \\
& \leq\left\|\left[I+\Delta t \theta M\left(\overrightarrow{\mathcal{R}}^{n}\right)\right]^{-1}\right\|_{\infty} \|\left[I-(1-\theta) \Delta t M(\overrightarrow{\mathcal{R}})\left\|_{\infty}\right\| \overrightarrow{\mathcal{R}}^{n} \|_{\infty}\right. \\
& \leq\left\|\overrightarrow{\mathcal{R}}^{n}\right\|_{\infty}
\end{aligned}
$$

In the presence of the linear source term with $D \geq 0$, we obtain the following stability estimate:

$$
\left\|\overrightarrow{\mathcal{R}}^{n+1}\right\|_{\infty} \leq\left(1+\Delta t\|D\|_{\infty}\right)\left\|\overrightarrow{\mathcal{R}}^{n}\right\|_{\infty} \leq\left(1+\Delta t \max _{j}\left(D_{j j}\right)\right)\left\|\overrightarrow{\mathcal{R}}^{n}\right\|_{\infty}
$$

This result is expected because the differential equation admits growth of this sort in the presence of the source term (with positive coefficient).

We now turn to the actual discretization of the individual terms. For convenience we rewrite the diffusion terms (assuming $\nu$ is constant)

$$
\left(\nu+\frac{\nu_{t}}{\sigma_{\epsilon}}\right) \nabla^{2}\left(\nu \widetilde{R}_{T}\right)-\frac{1}{\sigma_{\epsilon}}\left(\nabla \nu_{t}\right) \cdot \nabla\left(\nu \widetilde{R}_{T}\right)=2\left(\nu+\frac{\nu_{t}}{\sigma_{\epsilon}}\right) \nabla^{2}\left(\nu \widetilde{R}_{T}\right)-\frac{1}{\sigma_{\epsilon}} \nabla \cdot \nu_{t} \nabla\left(\nu \widetilde{R}_{T}\right)
$$

Using this identity, we rewrite equation (13) $\left(S^{2}=\left(\frac{\partial U_{i}}{\partial x_{j}}+\frac{\partial U_{j}}{\partial x_{i}}\right) \frac{\partial U_{i}}{\partial x_{j}}\right)$

$\frac{\partial\left(\nu \widetilde{R}_{T}\right)}{\partial t}+\mathbf{V} \cdot \nabla\left(\nu \widetilde{R}_{T}\right)=2\left(\nu+\frac{\nu_{t}}{\sigma_{\epsilon}}\right) \nabla^{2}\left(\nu \widetilde{R}_{T}\right)-\frac{1}{\sigma_{\epsilon}} \nabla \cdot \nu_{t} \nabla\left(\nu \widetilde{R}_{T}\right)+\left(c_{\epsilon_{2}} f_{2}-c_{\epsilon_{1}}\right) \sqrt{c_{\mu} f_{\mu} f_{3}} S \widetilde{R}_{T}$

We first approximate the advective terms by using a standard, first-order accurate upwind approximation $\left(u^{ \pm}=(u \pm|u|) / 2, v^{ \pm}=(v \pm|v|) / 2\right)$ :

$$
\begin{aligned}
\mathbf{V} \cdot \nabla\left(\nu \widetilde{R}_{T}\right) & \approx \alpha_{a}^{x} \mathcal{R}_{j+1, k}+\beta_{a}^{x} \mathcal{R}_{j, k}+\gamma_{a}^{x} \mathcal{R}_{j-1, k} \\
& +\alpha_{a}^{y} \mathcal{R}_{j, k+1}+\beta_{a}^{y} \mathcal{R}_{j, k}+\gamma_{a}^{y} \mathcal{R}_{j, k-1}
\end{aligned}
$$

where

$$
\begin{array}{lll}
\alpha_{a}^{x}=\frac{1}{\Delta x} u_{j, k}^{-}, & \gamma_{a}^{x}=-\frac{1}{\Delta x} u_{j, k}^{+}, & \beta_{a}^{x}=-\left(\alpha_{a}^{x}+\gamma_{a}^{x}\right) \\
\alpha_{a}^{y}=\frac{1}{\Delta y} v_{j, k}^{-}, & \gamma_{a}^{y}=-\frac{1}{\Delta y} v_{j, k}^{+}, & \beta_{a}^{y}=-\left(\alpha_{a}^{y}+\gamma_{a}^{y}\right)
\end{array}
$$

Note that this discretization automatically satisfies both properties 1 and 2 mentioned previously. 
The diffusive/antidiffusive terms are approximated by central differencing:

$$
\begin{aligned}
2\left(\nu+\frac{\nu_{t}}{\sigma}\right) \nabla^{2}\left(\nu \tilde{R}_{T}\right) \approx 2\left(\nu+\frac{\nu_{t}}{\sigma}\right)_{j, k} & {\left[\frac{\mathcal{R}_{j+1, k}-2 \mathcal{R}_{j, k}+\mathcal{R}_{j-1, k}}{\Delta x^{2}}\right.} \\
& \left.+\frac{\mathcal{R}_{j, k+1}-2 \mathcal{R}_{j, k}+\mathcal{R}_{j, k-1}}{\Delta y^{2}}\right] \\
\nabla \cdot \frac{\nu_{t}}{\sigma} \nabla\left(\nu \widetilde{R}_{T}\right) \approx & \frac{1}{\sigma}\left[\frac{\left(\nu_{t}\right)_{j+1 / 2, k}\left(\mathcal{R}_{j+1, k}-\mathcal{R}_{j, k}\right)-\left(\nu_{t}\right)_{j-1 / 2, k}\left(\mathcal{R}_{j, k}-\mathcal{R}_{j-1, k}\right)}{\Delta x^{2}}\right. \\
& \left.+\frac{\left(\nu_{t}\right)_{j, k+1 / 2}\left(\mathcal{R}_{j, k+1}-\mathcal{R}_{j, k}\right)-\left(\nu_{t}\right)_{j, k-1 / 2}\left(\mathcal{R}_{j, k}-\mathcal{R}_{j, k-1}\right)}{\Delta y^{2}}\right]
\end{aligned}
$$

By combining these two expressions we obtain

$$
\begin{aligned}
2\left(\nu+\frac{\nu_{t}}{\sigma}\right) \nabla^{2}\left(\nu \widetilde{R}_{T}\right)-\nabla \cdot \frac{\nu_{t}}{\sigma} \nabla\left(\nu \widetilde{R}_{T}\right) & \approx \alpha_{d}^{x} \mathcal{R}_{j+1, k}+\beta_{d}^{x} \mathcal{R}_{j, k}+\gamma_{d}^{x} \mathcal{R}_{j-1, k} \\
& +\alpha_{d}^{y} \mathcal{R}_{j, k+1}+\beta_{d}^{y} \mathcal{R}_{j, k}+\gamma_{d}^{y} \mathcal{R}_{j, k-1}
\end{aligned}
$$

where

$$
\begin{array}{ll}
\alpha_{d}^{x}=\frac{1}{\Delta x^{2}}\left[2\left(\nu+\frac{\nu_{t}}{\sigma}\right)_{j, k}-\left(\frac{\nu_{t}}{\sigma}\right)_{j+1 / 2, k}\right] & \gamma_{d}^{x}=\frac{1}{\Delta x^{2}}\left[2\left(\nu+\frac{\nu_{t}}{\sigma}\right)_{j, k}-\left(\frac{\nu_{t}}{\sigma}\right)_{j-1 / 2, k}\right] \\
\alpha_{d}^{y}=\frac{1}{\Delta y^{2}}\left[2\left(\nu+\frac{\nu_{t}}{\sigma}\right)_{j, k}-\left(\frac{\nu_{t}}{\sigma}\right)_{j, k+1 / 2}\right] & \gamma_{d}^{y}=\frac{1}{\Delta y^{2}}\left[2\left(\nu+\frac{\nu_{t}}{\sigma}\right)_{j, k}-\left(\frac{\nu_{t}}{\sigma}\right)_{j, k-1 / 2}\right] \\
\beta_{d}^{x}=-\left(\alpha_{d}^{x}+\gamma_{d}^{x}\right) & \\
\beta_{d}^{y}=-\left(\alpha_{d}^{y}+\gamma_{d}^{y}\right)
\end{array}
$$

Note that in this form we do not have automatic satisfaction of property 1 which would require that both $\alpha$ and $\gamma$ be positive coefficients (property 2 is automatically satisfied). It is important to realize that if these coefficients become negative this effect is entirely a result of poor grid resolution. To see this, assume a smooth variation of $\nu_{t}$ and expand $\nu_{t}$ in a Taylor series. For example we have that

$$
\left(\nu_{t}\right)_{j+1 / 2, k}=\left(\nu_{t}\right)_{j, k}+O(\Delta x)
$$

and

$$
\alpha_{d}^{x}=\frac{1}{\Delta y^{2}}\left[2 \nu+\left(\frac{\nu_{t}}{\sigma}\right)_{j, k}+O(\Delta x)\right]
$$

From this equation it is clear that this coefficient is guaranteed positive for small enough $\Delta x$. If we assume that $\nu_{t}>>$, we obtain the following restriction for non-negativity of $\alpha$ and $\gamma$ :

$$
\begin{array}{ll}
\left(\nu_{t}\right)_{j+1, k} \leq 3\left(\nu_{t}\right)_{j, k}, & \left(\nu_{t}\right)_{j-1, k} \leq 3\left(\nu_{t}\right)_{j, k} \\
\left(\nu_{t}\right)_{j, k+1} \leq 3\left(\nu_{t}\right)_{j, k}, & \left(\nu_{t}\right)_{j, k-1} \leq 3\left(\nu_{t}\right)_{j, k}
\end{array}
$$


In our implementation of the algorithm we strictly enforce this condition. Whenever these conditions are violated we limit the amount of anti-diffusion added so as to maintain positivity. Keep in mind that the better remedy, however, is usually grid refinement.

We conclude this section with a final remark concerning grid-resolution requirements for the one-equation model. Because the variable $\widetilde{R}_{T}$ was designed to behave linearly in the near-wall region for zero pressure gradient boundary layers (as previously shown in Figure 3 and equation (18)), we typically only require a mesh wall spacing comparable to the Baldwin-Lomax model $\left(y^{+}<3.5\right)$. This limit is required for accurately estimating wall shear which is used in the present implementation to determine $y^{+}$in the damping functions. This is a drastic improvement over near-wall formulations of the $k-\epsilon$ equations which typically require mesh wall spacing less than $y^{+}=0.2$ as mentioned in reference 9 . This removes from the flow solver much of the stiffness resulting from the extremely fine meshes which must be used in the near-wall formulations of the $k-\epsilon$ model. In figure 16 we plot the law of the wall for the flat plate using several mesh wall spacings $0.5<y_{\text {wall }}^{+}<3.1$ to demonstrate the solution independence with wall spacing.

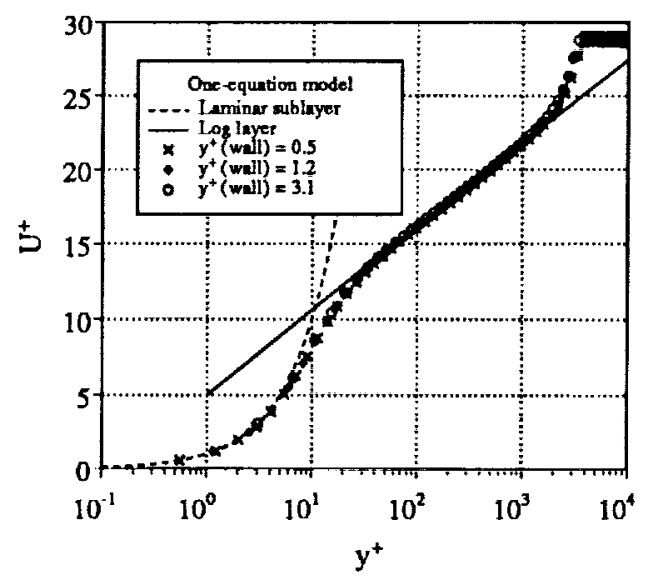

Figure 16. Sensitivity of boundary layer to wall spacing.

\section{CONCLUSIONS}

A one-equation turbulence model has been introduced that avoids the necessity for finding an algebraic length scale. The preliminary results presented indicate that the model has predictive and numerical properties of sufficient interest to merit further investigation and refinement. 


\section{APPENDIX - SUMMARY OF THE ONE-EQUATION MODEL}

This appendix gives a complete summary of the one-equation model. We begin with the field equation for $\widetilde{R}_{T}$ :

$$
\frac{D\left(\nu \widetilde{R}_{T}\right)}{D t}=\left(c_{\epsilon_{2}} f_{2}-c_{\epsilon_{1}}\right) \sqrt{\nu \widetilde{R}_{T} P}+\left(\nu+\frac{\nu_{t}}{\sigma_{\epsilon}}\right) \nabla^{2}\left(\nu \widetilde{R}_{T}\right)-\frac{1}{\sigma_{\epsilon}}\left(\nabla \nu_{t}\right) \cdot \nabla\left(\nu \widetilde{R}_{T}\right)
$$

In this equation, we use the following functions:

$$
\begin{aligned}
\frac{1}{\sigma_{\epsilon}} & =\left(c_{\epsilon_{2}}-c_{\epsilon_{1}}\right) \sqrt{c_{\mu}} / \kappa^{2} \\
\nu_{t} & =c_{\mu}\left(\nu \widetilde{R}_{T}\right) D_{1} D_{2} \\
\mu_{t} & =\rho \nu_{t} \\
D_{1} & =1-\exp \left(-y^{+} / A^{+}\right) \\
D_{2} & =1-\exp \left(-y^{+} / A_{2}^{+}\right) \\
P & =\nu_{t}\left(\frac{\partial U_{i}}{\partial x_{j}}+\frac{\partial U_{j}}{\partial x_{i}}\right) \frac{\partial U_{i}}{\partial x_{j}}-\frac{2}{3} \nu_{t}\left(\frac{\partial U_{k}}{\partial x_{k}}\right)^{2} \\
f_{2}\left(y^{+}\right) & =\frac{c_{\epsilon_{1}}}{c_{\epsilon_{2}}}+\left(1-\frac{c_{\epsilon_{1}}}{c_{\epsilon_{2}}}\right)\left(\frac{1}{\kappa y^{+}}+D_{1} D_{2}\right)\left(\sqrt{D_{1} D_{2}}\right. \\
& \left.+\frac{y^{+}}{\sqrt{D_{1} D_{2}}}\left(\frac{1}{A^{+}} \exp \left(-y^{+} / A^{+}\right) D_{2}+\frac{1}{A_{2}^{+}} \exp \left(-y^{+} / A_{2}^{+}\right) D_{1}\right)\right)
\end{aligned}
$$

For all calculations we have used the following constants:

$$
\begin{aligned}
\kappa & =0.41, & c_{\epsilon_{1}}=1.2, & c_{\epsilon_{2}}=2.0 \\
c_{\mu} & =0.09, & A^{+}=26, & A_{2}^{+}=10
\end{aligned}
$$

We also recommend the following boundary conditions for (35):

1. Solid Walls: Specify $\widetilde{R}_{T}=0$.

2. Inflow $(\mathbf{V} \cdot \mathbf{n}<0)$ : Specify $\tilde{R}_{T}=\left(\tilde{R}_{T}\right)_{\infty}<1$.

3. Outflow $(\mathbf{V} \cdot \mathbf{n}>0)$ : Extrapolate $\widetilde{R}_{T}$ from interior values. 


\section{REFERENCES}

1. Baldwin, B.S.; and Lomax, H.L.: Thin Layer Approximation and Algebraic Model for Separated Turbulent Flows. AIA A Paper 78-257, January 1978.

2. Cebeci, T.: Calculation of Compressible Turbulent Boundary-Layers with Heat and Mass Transfer. AIA A Journal, vol. 9, no. 6, 1971, pp. 1091-1097.

3. Maksymiuk, C.M.; and Pulliam, T.H.: Viscous Transonic Airfoil Workshop Results Using Arc2D. AIA A Paper 87-0415, January 1987.

4. Johnson, D.A.; and King, L.S.: A Mathematically Simple Turbulence Closure Model for Attached and Separated Turbulent Boundary Layers. AIA A Journal, vol. 23, no. 11, 1985, pp. 1684-1692.

5. King, L.S.: A Comparsion of Turbulence Closure Models for Transonic Flows about Airfoils. AIAA Paper 87-0418, January 1987.

6. Coakley, T.J.: Numerical Simulation of Viscous Transonic Airfoil Flows. AIAA Paper 87-0416, January 1987.

7. Pulliam, T.H.; Jespersen, D.J.; and Barth, T.J.: Navier-Stokes Computations for Circulation Controlled Airfoils. AIA A Paper 85-1587, July 1985.

8. Sugavanum, A.: Evaluation of Low Reynolds Number Turbulence Models for Attached and Separated Flows. AIA A Paper 85-0375, January 1985.

9. Patel, V.C.; Rodi, W.; and Scheurer, G.: Turbulence Models for Near-Wall and Low Reynolds Number Flows: A Review. AIA A Journal, vol. 23, no. 9, 1985, pp. 1308-1319.

10. Mansour, N.N.; Kim, J.; and Moin, P.: Near-Wall $k-\epsilon$ Turbulence Modeling. AIAA Journal, vol. 27, no. 8, 1989, pp. 1068-1073.

11. Hopkins, E.J.; and Inouye, M.: An Evaluation of Theories for Predicting Turbulent Skin Friction and Heat Transfer on Flat Plates at Supersonic and Hypersonic Mach Numbers. AIA A Journal, vol. 9, no. 6, June 1971, pp. 993-1003.

12. Rubesin, M.W.; and Horstman, C.C.: Supersonic Flow over a Flat Plate. The 198081 AFOSR-HTTM-Stanford Conference on Complex Turbulent Flows, vol. 1, S.J. Kline, B. Cantwell, and G.M. Lilley, eds., Thermo Sciences Division, Mechanical Engineering Department, Stanford University, Stanford, California, 1981, pp. 369-377.

13. Tennekes, H.; and Lumley, J.L.: A First Course in Turbulence. The MIT Press, Cambridge, Mass. and London, England, 1972.

14. Townsend, A.A.: The Structure of Turbulent Shear Flow. Second ed., Cambridge University Press, 1976.

15. Barth, T.J.; and Lomax, H.L.: Algorithm Development. NASA CP-2454, 1987. 



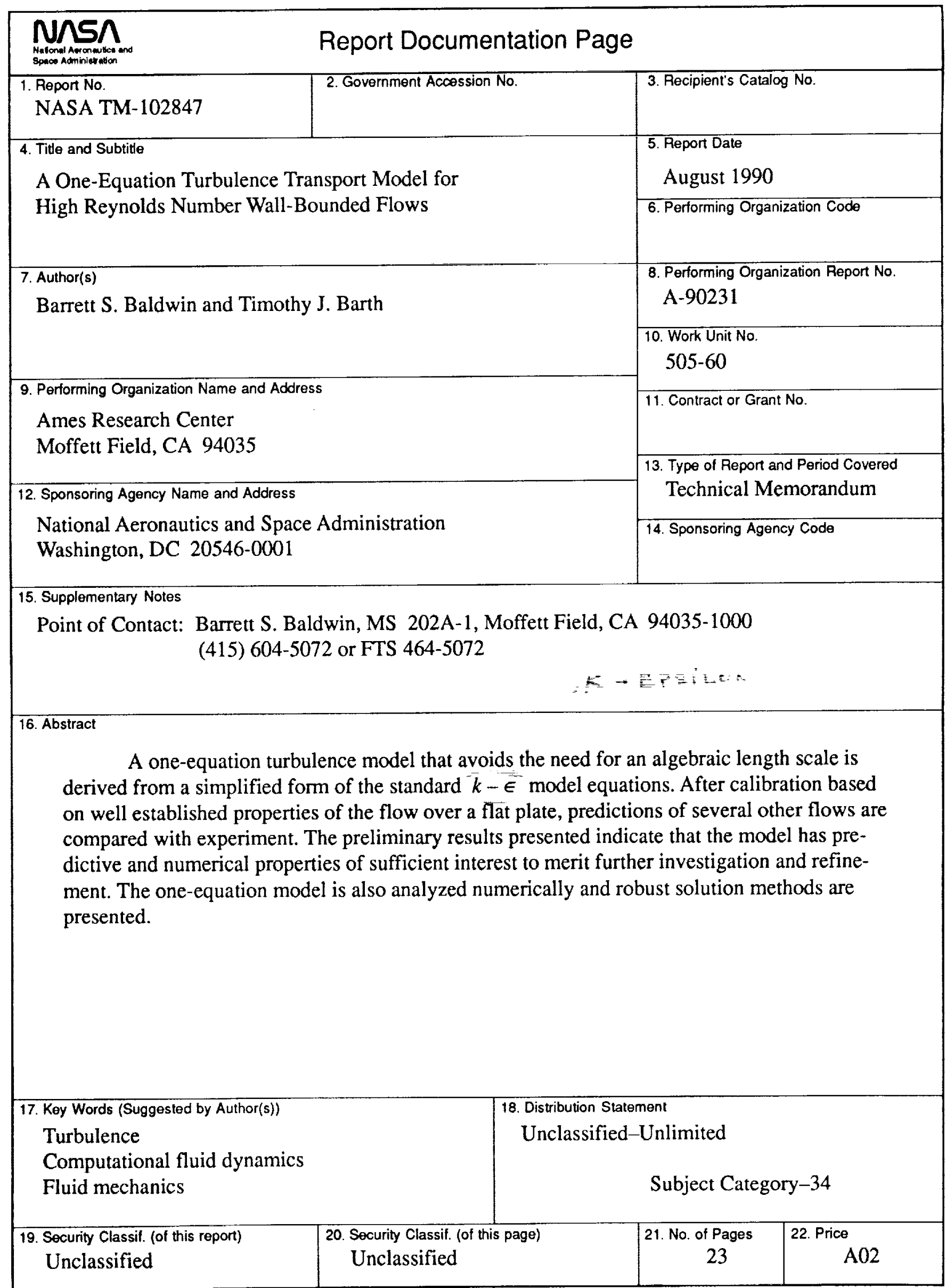

